\title{
Comprehensive study on the molecular prevalence and seasonal and age distribution of Mycobacterium avium subspecies paratuberculosis in raw milk and traditional dairy products
}

\author{
Rasoul Shirvani ${ }^{1,2}$, Ebrahim Rahimi ${ }^{1,2}$, Amir Shakerian' ${ }^{1}$ and Hassan Momtaz ${ }^{3}$ \\ Received: 22 August 2019 / Accepted: 19 September 2019 / Published online: 31 December 2019 \\ (C) Indian Dairy Association (India) 2019
}

\begin{abstract}
Due to the high consumption rate of milk and dairy products, they should have a high microbial quality. Maycobacterium avium subspecies paratuberclosis is one of the emerging zoonotic bacteria with high economic importance. The present study was done to assess the prevalence of the MAP in different types of raw milk and traditional dairy samples. Seven-hundred and eighty-five raw milk and traditional dairy samples were collected. DNA was extracted using a DNA extraction kit. Detection of MAP was done using the $I S 900$ nested-PCR method. Eighty-eight out of 785 (11.21\%) raw milk and traditional dairy product samples harbored MAP. Prevalence of MAP in raw milk and traditional dairy samples were $12.38 \%$ and $8.84 \%$, respectively. Raw buffalo (25\%) milk samples had the highest prevalence of MAP, while raw donkey $(6.66 \%)$ had the lowest. Traditional cheese (24\%) samples had the highest prevalence of MAP, while traditional kashk (2.50\%) had the lowest. Seasonal and age distributions were also observed for the prevalence of MAP. Raw milk and traditional dairy samples may be the sources of MAP transmission into the human population. Marked seasonality and age distribution were seen for the prevalence of MAP. Further studies are required to find more information about MAP infection in raw milk and traditional dairy products.
\end{abstract}

\footnotetext{
${ }^{1}$ Department of Food Hygiene, Faculty of Veterinary Medicine, Shahrekord Branch, Islamic Azad University, Shahrekord, Iran

${ }^{2}$ Research Center of Nutrition and Organic Products, Shahrekord Branch, Islamic Azad University, Shahrekord, Iran
${ }^{3}$ Department of Microbiology, Faculty of Basic Sciences, Shahrekord Branch, Islamic Azad University, Shahrekord, Iran
Ebrahim Rahimi $(\bowtie)$
Department of Food Hygiene,
Faculty of Veterinary Medicine, Shahrekord Branch, Islamic Azad University, Shahrekord, Iran

Email address: Ebrahimrahimi55@yahoo.com
}

Keywords: Age distribution, Maycobacterium avium subspecies paratuberclosis, Raw milk, Seasonal distribution, Traditional dairy

\section{Introduction}

Milk and dairy products are full from valuable nutritional factors such as proteins, lipids, minerals, and vitamins. There are several kinds of dairy products such as cheese, cream, butter, yoghurt, and ice cream with high beneficial effects on human health (Momtaz et al. 2012; Rahimi et al. 2014a; Ranjbar et al. 2018a; Ranjbar et al. 2018b; Safarpoor Dehkordi et al. 2013a; Safarpoor Dehkordi et al. 2014b). Kashk and dough are two important traditional dairy products in Iran and some other parts of the world. Kashk comes in liquid or dried forms and is traditionally made with the milk left over from cheese-making. Dough is a popular salty yogurt-based beverage amongst an Iranian people (Momtaz et al. 2012; Rahimi et al. 2014a; Ranjbar et al. 2018a; Ranjbar et al. 2018b; Safarpoor Dehkordi et al. 2013a; Safarpoor Dehkordi et al. 2014b). Based on the high consumption rate of milk and dairy products, it is important to ensure from the microbial quality of these types of food samples (Momtaz et al. 2012; Rahimi et al. 2014a; Ranjbar et al. 2018a; Ranjbar et al. 2018b; Safarpoor Dehkordi et al. 2013a; Safarpoor Dehkordi et al. 2014b). However, the microbial quality of raw milk and traditional dairy products has been decreased and several foodborne infections have been occurred (Atapoor et al. 2014; Ghorbani et al. 2016; Hasanpour Dehkordi et al. 2017; Hemmatinezhad et al. 2015; Madahi et al. 2014; Momtaz et al. 2013a; Momtaz et al. 2013b; Nejat et al. 2015; Rahimi et al. 2014b; Ranjbar et al. 2017; Safarpoor Dehkordi et al. 2017; Safarpoor Dehkordi et al. 2013b; Safarpoor Dehkordi et al. 2014a; Safarpoor Dehkordi et al. 2012).

Mycobacterium avium (M. avium) subsp. paratuberculosis (MAP) is the causal agent of Johne's disease (a chronic granulomatous enteritis of cattle and other ruminants) and chronic infectious enteritis and especially Paratuberculosis of domestic and wild ruminants. Paratuberculosis is characterized by a long incubation period, weight loss, diarrhea, progressive cachexia, and death (Chamberlin et al. 2001; Manning 2001). Furthermore, MAP has also been isolated in intestinal tissues of Crohn's disease (a chronic inflammatory condition of the gut) patients, 
thus giving rise to a hypothesis of there being a possible link between these two diseases (Chamberlin et al. 2001; Manning 2001). Milk plays an important role in transmission of infection and above all the excretion via milk is viewed with caution since raw milk may be a possible source of human infection (Chamberlin et al. 2001; Gill et al. 2011; Grant 2003; Manning 2001). It has long been suggested that MAP may cause or be otherwise involved in Crohn's disease, but the available evidence of this involvement has been inconclusive. Additionally, the concern about presence of MAP in milk and dairy products is about its heat resistance nature and also its controversial role in occurrence of Crohn's disease in human (Chamberlin et al. 2001; Gill et al. 2011; Grant 2003; Manning 2001).

Traditionally, MAP culture from ruminant's milk is a low sensitive, time consuming and dangerous diagnostic method (Chamberlin et al. 2001; Gill et al. 2011; Grant 2003; Manning 2001). Highly sensitive molecular techniques such as different types of Polymerase Chain Reaction (PCR) have greatly contributed to identify MAP strains in food samples with animal origins and especially milk (Pillai \& Jayarao 2002). As yet there is no literature concerning the detection of MAP by PCR in raw milk and traditional dairy products in Iran. Therefore, the present investigation was done to study the prevalence rate and seasonal and age distributions of MAP in raw bovine, ovine, caprine, buffalo, camel and donkey milk and traditional cheese, dough, kashk, yoghurt, butter and cream dairy product samples using the nested-PCR reaction.

\section{Materials and Methods}

\section{Ethics approval and consent to participate}

The study was approved by the Ethical Council of Research of the Faculty of Veterinary Medicine, Shahrekord Branch, Islamic Azad University, Shahrekord, Iran. Verification of this research project and the licenses related to sampling process were approved by the Prof. Ebrahim Rahimi and Prof. Amir Shakerian.

\section{Samples collection}

From June 2016 to June 2017, a total of 785 various types of dairy samples including raw bovine $(n=120)$, ovine $(n=120)$, caprine $(n=110)$, camel $(n=85)$, buffalo $(n=60)$ and donkey $(n=30)$ raw milk samples and traditional cheese $(n=50)$, yoghurt $(n=40)$, dough $(n=50)$, cream $(n=40)$, butter $(n=40)$ and kashk $(n=40)$ dairy product samples were randomly collected from different parts of Iran. Before collection, teats were thoughtfully cleansed with alcohol to avoid samples contamination with the skin. Milk $(40 \mathrm{~mL})$ was collected in a sterile $50-\mathrm{mL}$ centrifuge tube from the 4 quarters by milking, discarding the first 10-15 mL. Samples were immediately transferred to the Food Hygiene Research Center of the Islamic Azad University of Shahrekord in cooler with icepacks. All samples showed normal physical characters including odor, color and consolidation.

\section{Milk preparation and DNA extraction}

Prior to DNA extraction, milk samples were centrifuged at $1000 \mathrm{~g}$ for $15 \mathrm{~min}$ and the supernatant discarded. The resultant pellet was washed thrice in phosphate buffer saline (PBS, pH 7.3) and centrifuged at $500 \mathrm{~g}$ for $15 \mathrm{~min}$. the pellet was suspended in $1 \mathrm{~mL}$ of PBS, centrifuged and resuspended in $100 \mathrm{~mL}$ of $0.2 \mathrm{~N} \mathrm{NaOH}$. DNA extracted using a commercial kit (DNeasy tissue kit, Qiagen, Hilden, Germany) preceded by an enzymatic digestion with lysozyme buffer (lysozyme $18 \mathrm{mg} / \mathrm{ml}, 15 \mathrm{mM}$ Tris- $\mathrm{HCl}$ pH 8.0, 1 mM EDTA and $1 \%$ Triton X100) and proteinase K (Qiagen, Hilden, Germany). Procedure was done rendering to the manufacturer's guidelines. Purity (A260/A280) and concentration of extracted DNA were then checked (NanoDrop, Thermo Scientific, Waltham, MA, USA). The truth of the DNA was assessed on a $2 \%$ agarose gel stained with ethidium bromide $(0.5 \mu \mathrm{g} / \mathrm{mL})$ (Thermo Fisher Scientific, St. Leon-Rot, Germany).

\section{Nested PCR-based detection of M. avium subsp. paratuberculosis}

M. avium subsp. paratuberculosis was detected using the specific nested-PCR reaction according to method described previously (Englund et al. 1999; Nebbia et al. 2006). Four primers were selected from the $M$. avium subsp. paratuberculosis specific insertion sequence IS900 (Accession No. X16293 in GenBank). Table 1 represents the list of primers used in primary and nested PCR reactions.

The first and the second amplification reaction were optimized individually regarding the concentration of $\mathrm{MgCl} 2$, nucleotides, primer, and Taq DNA polymerase as well as the annealing temperature and number of cycles in the amplification. In the reactions $1 \mathrm{pg}$ to $50 \mathrm{pg}$ DNA from milk and traditional dairy samples were used. Finally, the $50-\mathrm{mL}$ reaction mixture contained $60 \mathrm{mM}$ Tris- $\mathrm{HCl}$ buffer (pH 8.8), $2.0 \mathrm{mM} \mathrm{MgCl} 2,0.2 \mathrm{mM}$ of each of the four dNTPs (Thermo Fisher Scientific, St. Leon-Rot, Germany), 10 pmol of each of the primers, 0.5 U Taq DNA

Table 1 List of primers used for detection of the M. avium subsp. paratuberculosis in raw milk and traditional dairy product samples

\begin{tabular}{lllc}
\hline Target gene & Primer Sequence (5'-3') & PCR product & Size of product (bp) \\
\hline$I S 900$ & GTTCGGGGCCGTCGCTTAGG & Amplicon 1 & 400 \\
$I S 900$ & GAGGTCGATCGCCCACGTGA & & 229 \\
$I S 900$ & CCGCTAATTGAGAGATGCGATTGG & Amplicon 2 & \\
$I S 900$ & AATCAACTCCAGCAGCGCGGCCTCG & & \\
\hline
\end{tabular}


polymerase, and $5 \mathrm{~mL}$ DNA. A positive control, $250 \mathrm{fg}$ DNA from $M$. avium subsp. paratuberculosis strain Linda, and a negative control, sterile water (Thermo Fisher Scientific, St. Leon-Rot, Germany), were included in each amplification set-up. The reaction mixtures were overlaid with two drops of mineral oil, centrifuged briefly and placed in a PCR thermal cycler (Eppendorf Co., Hamburg, Germany) programmed for $94^{\circ} \mathrm{C}$ for $3 \mathrm{~min}$, and then $94^{\circ} \mathrm{C}$ for $1 \mathrm{~min}, 63^{\circ} \mathrm{C}$ for $1 \mathrm{~min}$, and $72^{\circ} \mathrm{C}$ for $1 \mathrm{~min}$, the last three steps being repeated sequentially for 35 cycles. The second PCR was performed in the same way as the first PCR, but with a 5-mL sample from the first PCR reaction (Englund et al. 1999; Nebbia et al. 2006).

\section{Gel electrophoresis}

Amplified samples were analyzed by electrophoresis (120 V/208 $\mathrm{mA}$ ) in $2.5 \%$ agarose gel. The gel was stained with $0.1 \%$ ethidium bromide $(0.4 \mu \mathrm{g} / \mathrm{ml})$. The UVI doc gel documentation systems (Grade GB004, Jencons PLC, London, UK) was applied for analysis of images.

\section{Statistical analysis}

Statistics were subjected to Microsoft office Excel (version 15; Microsoft Corp., Redmond, WA, USA). Statistical analysis was performed by means of the SPSS 21.0 statistical software (SPSS Inc., Chicago, IL, USA). Chi-square test and Fisher's exact twotailed test were applied to measure any significant relationship. $P$ value $<0.05$ was considered as statistical significant level.

\section{Results and Discussion}

\section{Nested-PCR amplification}

MAP is responsible for occurrence of dangerous pathogenic diseases such as paratuberculosis and Johne's and Crohn's diseases. Foods with animal origins and especially raw milk and traditional dairy products paly an emerging role in transmission of MAP to human population. MAP is present in domesticated ruminants in most regions of the world. Determination of the MAP infection status of individual animals or herds can be difficult. Consequently, estimates of the prevalence of MAP in animals and herds also is difficult (Gill et al. 2011; Grant 2003). Additionally, scarce data have been conducted on detection of MAP from raw milk of naturally infected animals and also traditional dairy products (Gill et al. 2011; Grant 2003). Thus, the present study was done to assess the prevalence of MAP in raw milk of naturally infected animals and also traditional dairy products.

The present study was done to assess the prevalence rate and seasonal and age distributions of the MAP in different types of raw milk and traditional dairy product samples. Figure 1 represents the results of the PCR gel electrophoresis for the nested-PCR reaction used for detection of the IS900 gene of the MAP.

\section{Prevalence of MAP in studied dairy}

Table 2 represents the prevalence of MAP in different types of raw milk and traditional dairy product samples. Eighty-eight out of $785(11.21 \%)$ raw milk and traditional dairy product samples harbored MAP. Sixty-five out of 525 (12.38\%) raw milk samples and twenty-three out of $260(8.84 \%)$ traditional dairy product

Table 2 Prevalence of $M$. avium subsp. paratuberculosis in different types of raw milk and traditional dairy product samples

\begin{tabular}{llll}
\hline Types of samples & No. samples collected & $\begin{array}{l}\text { N (\%) samples positive for } \\
\text { M. avium subspecies } \\
\text { paratuberculosis }\end{array}$ & P value \\
\hline Raw bovine milk & 120 & $19(15.83)$ & \\
Raw ovine milk & 120 & $12(10)$ & \\
Raw caprine milk & 110 & $9(8.18)$ & $P<0.05$ \\
Raw camel milk & 85 & $8(9.41)$ & \\
Raw buffalo milk & 60 & $15(25)$ & \\
Raw donkey milk & 30 & $2(6.66)$ & \\
Total raw milk & 525 & $65(12.38)$ & \\
Traditional cheese & 50 & $12(24)$ & \\
Traditional yoghurt & 40 & $2(5)$ & \\
Traditional dough & 50 & $2(4)$ & \\
Traditional cream & 40 & $3(7.50)$ & \\
Traditional Butter & 40 & $3(7.50)$ & \\
Traditional kashk & 40 & $1(2.50)$ & \\
Total traditional dairy & 260 & $23(8.84)$ & \\
products & & $88(11.21)$ & \\
Total & 785 &
\end{tabular}


Fig. 1 Results of the gel electrophoresis of the IS900 gene of the Mycobacterium avium subsp. paratuberculosis. A: the first step PCR amplification (400 bp) and B: The nested PCR amplification (229 bp). Lane 1: 100 bp ladder (Thermo Fisher Scientific, Germany), Lane 2: Positive control, Lane 3-6: Positive samples for the IS900 gene of the Mycobacterium avium subsp. paratuberculosis (400 bp in amplicon 1 and 229 bp in amplicon 2) and Lane7: Negative control

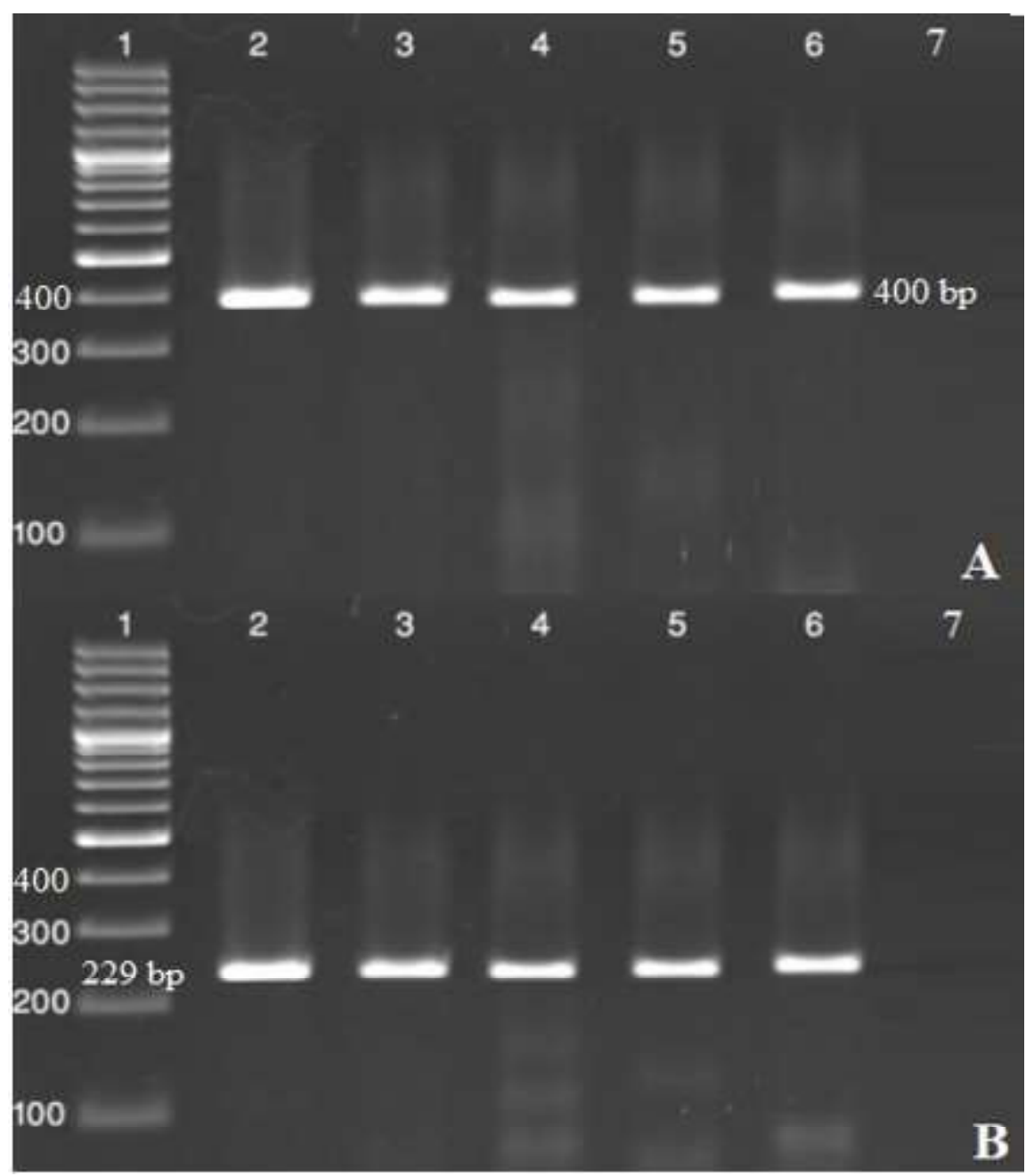

reveals that there is wide regional variation in MAP prevalence. For cattle, estimated animal prevalence have ranged from $<2$ to $<20 \%$, and estimated herd prevalence have ranged from $<10$ to $>50 \%$ (Adaska \& Anderson 2003; Mohan et al. 2009; Tiwari et al. 2006). In some regions, the prevalence of MAP in beef cattle is apparently about half the prevalence in dairy cattle (Good et al. 2009; Okura et al. 2010). Data on MAP infection of sheep and goats are limited, but prevalence of up to $20 \%$ in European herds have been suggested (Nielsen \& Toft 2009). Prevalence of MAP in milk of naturally infected cows collected from United States (Sweeney et al. 1992), Canada (Gao et al. 2009), Brazil (Carvalho et al. 2009), Mexico (Favila-Humara et al. 2010), Denmark (Giese $\&$ Ahrens 2000), Poland (Szteyn et al. 2008) and United Kingdom (Grant et al. 2002) had a range between 1\% to 71\%. Prevalence of MAP in milk of naturally infected sheeps and goats collected from European countries had a range of 1 to $100 \%$ (Djønne et al. 2003; Grant et al. 2001; Mühlherr et al. 2003; Nebbia et al. 2006). Prevalence of MAP in dairy product samples had a range of 1$78 \%$ al-around the world (Hruska et al. 2005; Ikonomopoulos et al. 2005; Shankar et al. 2010). 


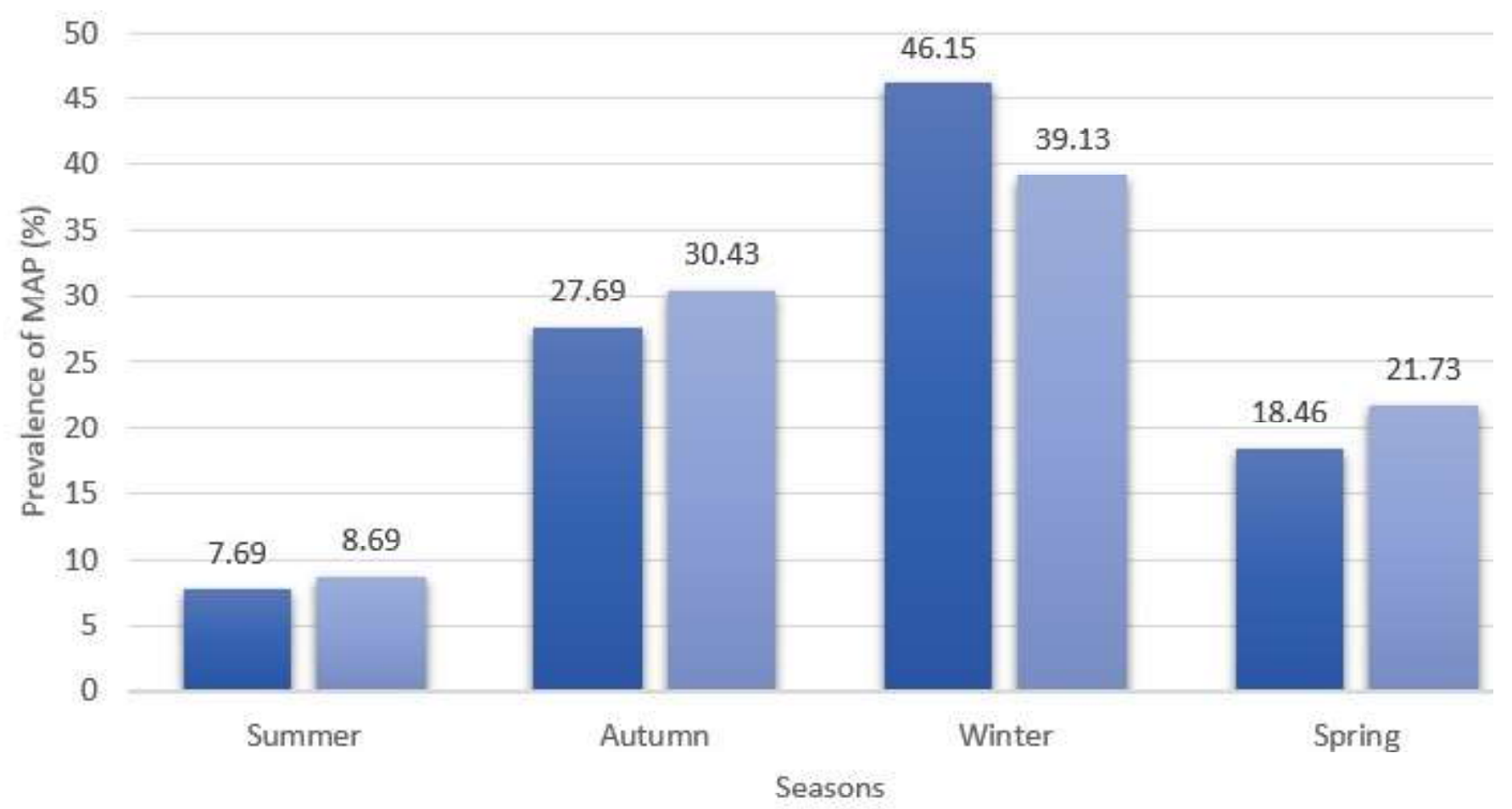

Raw milk $\quad$ Traditional dairy products

Fig. 2 Seasonal distribution of M. avium subsp. paratuberculosis in different types of raw milk and traditional dairy product samples

We found that raw buffalo (25\%) and raw bovine (15.83\%) milk and traditional cheese $(24 \%)$ samples had the highest prevalence of MAP. Higher tendency of the MAP bacteria to fat is may be probable reason for the higher prevalence of MAP in buffalo milk. In developed countries, most cheese is made from pasteurized milk. However, many types of cheese continue to be made from raw milk or milk that has been subjected to heating treatments less severe than those used for pasteurization. MAP obviously may be present in raw milk and can survive subpasteurization heating treatments. MAP is also resists against acidic $\mathrm{pH}$ and salt concentration present in cheese. Low prevalence of MAP in donkey milk samples is may be due to the fact that equines are not count as a real reservoir of the MAP. We also found that camel milk samples had the low prevalence of MAP. Low prevalence of MAP in kashk samples is may be due to the fact that kashk preparation needs high temperature which will decrease the numbers of MAP bacteria. Gilardoni et al. (2016) reported that the prevalence of MAP in milk in Argentina was $45.42 \%$. Nebbia et al. (2006) reported that the prevalence of MAP in milk in Italy was $44.80 \%$. Gerrard et al. (2018) reported that the prevalence of MAP in milk in England was 10.30\%.

\section{Age and seasonal distribution of MAP}

Table 3 represents the age distribution of MAP in different types of raw milk samples. We found that milk samples collected from
4-6 years old bovine (42.10\%), 2-4 years old ovine (41.66\%), 2-4 years old caprine $(44.44 \%), 2-6$ years old camel $(37.50 \%), 4-6$ years old buffalo (40\%) and 4-6 years old and older than 6 years old donkey $(50 \%)$ had the highest prevalence of MAP. Additionally, milk of younger than 2 years old animals had the lowest prevalence of MAP. Statistically significant difference was seen between age of animal species and prevalence of MAP $(P<0.05)$.

Figure 2 represents the seasonal distribution of MAP in raw milk and traditional dairy product samples. Raw milk samples which were collected through the winter season $(46.15 \%)$ had the highest prevalence of MAP, followed by autumn (27.69\%) and spring $(18.46 \%)$. Traditional dairy product samples which were collected through the winter season $(39.13 \%)$ had the highest prevalence of MAP, followed by autumn (30.43\%) and spring (21.73\%). Samples which were collected through the summer season had the lowest prevalence of MAP. Statistically significant differences were seen for the prevalence of MAP between cold and warm seasons $(P<0.05)$.

We also found that the prevalence of MAP in milk and dairy product samples follows the seasonal distribution. In the other hand, MAP bacteria had the highest prevalence in winter season. The main reason for this finding is the fact that transmission and dissemination of MAP is much higher in the winter than other 
Table 3 Age distribution of $M$. avium subsp. paratuberculosis in different types of raw milk samples

\begin{tabular}{|c|c|c|c|}
\hline $\begin{array}{l}\text { Types of samples (No. } \\
\text { positive) }\end{array}$ & Age (year) & $\begin{array}{l}\mathrm{N}(\%) \text { samples positive for } \\
\text { M. avium subspecies } \\
\text { paratuberculosis }\end{array}$ & $P$ value \\
\hline \multirow[t]{4}{*}{ Raw bovine milk(19) } & $<2$ & $1(5.26)$ & \\
\hline & $2-4$ & $4(21.05)$ & $P<0.05$ \\
\hline & $4-6$ & $8(42.10)$ & \\
\hline & $>6$ & $5(26.31)$ & \\
\hline \multirow[t]{4}{*}{ Raw ovine milk (12) } & $<2$ & $1(8.33)$ & \\
\hline & 24 & $5(41.66)$ & $P<0.05$ \\
\hline & $4-6$ & $3(25)$ & \\
\hline & $>6$ & $3(25)$ & \\
\hline \multirow[t]{4}{*}{ Raw caprine milk (9) } & $<2$ & - & \\
\hline & 24 & $4(44.44)$ & $P<0.05$ \\
\hline & $4-6$ & $3(33.33)$ & \\
\hline & $>6$ & $2(22.22)$ & \\
\hline \multirow[t]{4}{*}{ Raw camel milk (8) } & $<2$ & - & \\
\hline & $2-4$ & $3(37.50)$ & $P>0.05$ \\
\hline & $4-6$ & $3(37.50)$ & \\
\hline & $>6$ & $2(25)$ & \\
\hline \multirow[t]{4}{*}{ Raw buffalo milk (15) } & $<2$ & $1(6.66)$ & \\
\hline & $2-4$ & $4(26.66)$ & $P<0.05$ \\
\hline & $4-6$ & $6(40)$ & \\
\hline & $>6$ & $4(26.66)$ & \\
\hline \multirow[t]{4}{*}{ Raw donkey milk (2) } & $<2$ & - & \\
\hline & $2-4$ & - & $P>0.05$ \\
\hline & $4-6$ & $1(50)$ & \\
\hline & $>6$ & $1(50)$ & \\
\hline
\end{tabular}

tested seasons. Additionally, we found that milk of older animal species had the highest prevalence of MAP. Otherwise, milk of younger than 2 years old animal had the lowest prevalence of MAP. This finding is may be due to the long incubation period of MAP in animal species. MAP is responsible for Johne's disease and chronic infectious enteritis and especially Paratuberculosis of domestic and wild ruminants. Johne's disease is chronic granulomatous enteritis of cattle and other ruminants. Clinical practices revealed that occurrence of Johne's disease is so rare in younger than 2 years animal species (Adaska \& Anderson 2003; Good et al. 2009; Taylor et al. 1981).

Our results might suggest a MAP transmission risk via milk, even from apparently healthy animals. Infection of lambs and kids by this route is likely under normal breeding conditions. As reported for cattle, young animals are more susceptible to MAP infection than adults, with milk consumption from infected ewes being a major source of infection. Control measures similar to those already applied in cattle, such as sanitation, separating newborn calves from their mothers and subsequent artificial feeding, might be useful to small ruminants as well. Consumption of soft and hard cheese and other types of dairy products produced from un-pasteurized cow, sheep and goat milk is becoming increasingly important in Iran and other countries. This could increase the risk of MAP infection in humans. A link between MAP, Crohn's disease and consumption of dairy products from raw milk is still to be demonstrated (Chiodini 2003), however, even a remote public health risk should contribute to strengthen collaboration between veterinarians, public health officers, livestock breeders and dairy producers, as well as stimulate the development and subsequent routine use of more specific and sensitive diagnostic tools.

\section{Conclusions}

To put it in a nutshell, we identified a large numbers of MAP in raw bovine, ovine, caprine, buffalo, camel and donkey milk and also traditional cheese, yoghurt, cream, kashk, dough and butter dairy products. Raw buffalo and bovine milk samples and traditional cheese had the highest prevalence of MAP amongst tested samples. Therefore, raw buffalo and bovine milk and traditional cheese had the higher ability for transmission of MAP into the human populations. Additionally, prevalence of MAP bacteria was related to the seasonal distribution with higher prevalence in winter season. Additionally, prevalence of MAP bacteria was related to the age of animal species with higher prevalence in milk of older animals. To the best of our knowledge, this study is the most comprehensive description study on the 
prevalence of MAP in Iran. Although the incidence of MAP infection was relatively low (11.21\%), further studies should be conducted to get more information on MAP infection in raw bovine, ovine, caprine, buffalo, camel and donkey milk and also traditional cheese, yoghurt, cream, kashk, dough and butter dairy products, especially in areas where animals are kept close to human populations.

\section{Acknowledgements}

The authors would like to thank Dr. Manouchehr Momeni Shahraki at the Department of Food Hygiene and Quality Control, Shahrekord Branch, Islamic Azad University, Shahrekord, Iran for his important technical support.

\section{References}

Adaska JM, Anderson RJ (2003) Seroprevalence of Johne's-disease infection in dairy cattle in California, USA. Preventive Vet Med 60:255-261

Atapoor S, Dehkordi FS, Rahimi E (2014) Detection of Helicobacter pylori in various types of vegetables and salads. Jundishapur J Microb 7:e10013

Carvalho I, Silva Jr A, Campos V, Moreira M (2009) Detection of Mycobacterium avium subspecies paratuberculosis by polymerase chain reaction in bovine milk in Brazil. J Dairy Sci 92:5408-5410

Chamberlin W, Graham D, Hulten K (2001) Review article: Mycobacterium avium subsp. paratuberculosis as one cause of Crohn's disease. Aliment Pharm Ther 15:337-346

Chiodini RJ (2003) Twenty year later: where does it all stand? Paper presented at the Proceedings of the International Conference "Morbo di Crohn nell'uomo e paratubercolosi nei ruminanti, Rome, Italy, January. p 13-16.

Djønne B, Jensen M, Grant I, Holstad G (2003) Detection by immunomagnetic PCR of Mycobacterium avium subsp. paratuberculosis in milk from dairy goats in Norway. Vet Microbiol 92:135-143

Englund S, Ballagi-Pordány A, Bölske G, Johansson K-E (1999) Single PCR and nested PCR with a mimic molecule for detection of Mycobacterium avium subsp. paratuberculosis. Diagn Microbiol Infect Dis 33:163-171

Favila-Humara LC, Chávez-Gris GG, Carrillo-Casas EM, Hernández-Castro $\mathrm{R}$ (2010) Mycobacterium avium subsp. paratuberculosis detection in individual and bulk tank milk samples from bovine herds and caprine flocks. Foodborne Pathog Dis 7:351-355

Gao A, Odumeru J, Raymond M, Hendrick S, Duffield T, Mutharia L (2009) Comparison of milk culture, direct and nested polymerase chain reaction (PCR) with fecal culture based on samples from dairy herds infected with Mycobacterium avium subsp. paratuberculosis. Can J Vet Res 73:58-64

Gerrard ZE, et al. (2018) Survival of Mycobacterium avium subspecies paratuberculosis in retail pasteurised milk. Food Microbiol 74:5763

Ghorbani F, Gheisari E, Dehkordi FS (2016) Genotyping of vacA alleles of Helicobacter pylori strains recovered from some Iranian food items. Trop J Pharm Res 15:1631-1636

Giese SB, Ahrens P (2000) Detection of Mycobacterium avium subsp. paratuberculosis in milk from clinically affected cows by PCR and culture. Vet Microbiol 77:291-297
Gilardoni LR, et al. (2016) Mycobacterium paratuberculosis detection in cow's milk in Argentina by immunomagnetic separation-PCR. Braz J Microbiol 47:506-512

Gill C, Saucier L, Meadus W (2011) Mycobacterium avium subsp. paratuberculosis in dairy products, meat, and drinking water. J Food Prot 74:480-499

Good M, et al. (2009) Prevalence and distribution of paratuberculosis (Johne's disease) in cattle herds in Ireland. Irish Vet J 62:597

Grant I (2003) Mycobacterium paratuberculosis and milk. Acta Vet Scand 44:261-266

Grant I, O'Riordan L, Ball H, Rowe M (2001) Incidence of Mycobacterium paratuberculosis in raw sheep and goats' milk in England, Wales and Northern Ireland. Vet Microbiol 79:123-131

Grant IR, Ball HJ, Rowe MT (2002) Incidence of Mycobacterium paratuberculosis in bulk raw and commercially pasteurized cows' milk from approved dairy processing establishments in the United Kingdom. Appl Environ Microbiol 68:2428-2435

Hasanpour Dehkordi A, et al. (2017) One-year prevalence of antimicrobial susceptibility pattern of methicillin-resistant Staphylococcus aureus recovered from raw meat. Trop Biomed 34:396-404

Hemmatinezhad B, Khamesipour F, Mohammadi M, Safarpoor Dehkordi F, Mashak Z (2015) Microbiological Investigation of O Serogroups, Virulence Factors and Antimicrobial Resistance Properties of Shiga Toxin Producing Escherichia Coli Isolated from Ostrich, Turkey and Quail Meats. J Food Safety 35:491-500

Hruska K, Bartos M, Kralik P, Pavlik I (2005) Mycobacterium avium subsp. paratuberculosis in powdered infant milk: Paratuberculosis in cattle-the public health problem to be solved. Vet Med 50:327-335

Ikonomopoulos J, et al. (2005) Detection of Mycobacterium avium subsp. paratuberculosis in retail cheeses from Greece and the Czech Republic. Appl Environ Microbiol 71:8934-8936

Madahi H, Rostami F, Rahimi E, Dehkordi FS (2014) Prevalence of enterotoxigenic Staphylococcus aureus isolated from chicken nugget in Iran. Jundishapur J Microbiol 7:e10237.

Manning EJ (2001) Mycobacterium avium subspecies paratuberculosis: a review of current knowledge. J Zoo Wildlife Med 32:293-304

Mohan M, Duraisamy P, Praveena PE, Sivakumar P, Tripathi B, Singh N (2009) Prevalence paratub in cattle buffaloes. Indian Vet J 86:4-6

Momtaz H, Davood Rahimian M, Safarpoor Dehkordi F (2013a) Identification and characterization of Yersinia enterocolitica isolated from raw chicken meat based on molecular and biological techniques. J Appl Poult Res 22:137-145

Momtaz H, Dehkordi FS, Rahimi E, Asgarifar A, Momeni M (2013b) Virulence genes and antimicrobial resistance profiles of Staphylococcus aureus isolated from chicken meat in Isfahan province, Iran. J Appl Poult Res 22:913-921

Momtaz H, Safarpoor Dehkordi F, Taktaz T, Rezvani A, Yarali S (2012) Shiga toxin-producing Escherichia coli isolated from bovine mastitic milk: serogroups, virulence factors, and antibiotic resistance properties. Sci World J:618709.

Mühlherr JE, Zweifel C, Corti S, Blanco J, Stephan R (2003) Microbiological quality of raw goat's and ewe's bulk-tank milk in Switzerland. J Dairy Sci 86:3849-3856

Nebbia P, Robino P, Zoppi S, De Meneghi D (2006) Detection and excretion pattern of Mycobacterium avium subspecies paratuberculosis in milk of asymptomatic sheep and goats by Nested-PCR. Small Rum Res 66:116-120

Nejat S, Momtaz H, Yadegari M, Nejat S, Safarpour Dehkordi F, Khamesipour F (2015) Seasonal, geographical, age and breed distributions of equine viral arteritis in Iran. Kafkas Uni Vet Fak Derg 21:111-116

Nielsen SS, Toft N (2009) A review of prevalences of paratuberculosis in farmed animals in Europe. Prev Vet Med 88:1-14 
Okura H, Nielsen SS, Toft N (2010) Prevalence of Mycobacterium avium subsp. paratuberculosis infection in adult Danish non-dairy cattle sampled at slaughter. Prev Vet Med 94:185-190

Pillai S, Jayarao B (2002) Application of IS900 PCR for detection of Mycobacterium avium subsp. paratuberculosis directly from raw milk. J Dairy Sci 85:1052-1057

Rahimi E, Sepehri S, Dehkordi FS, Shaygan S, Momtaz H (2014a) Prevalence of Yersinia species in traditional and commercial dairy products in Isfahan Province, Iran. Jundishapur J Microbiol 7:e9249.

Rahimi E, Yazdanpour S, Dehkordi F (2014b) Detection of Toxoplasma gondii antibodies in various poultry meat samples using enzyme linked immuno sorbent assay and its confirmation by polymerase chain reaction. J Pure Appl Microbiol 8:421-7

Ranjbar R, Dehkordi FS, Shahreza MHS, Rahimi E (2018a) Prevalence, identification of virulence factors, O-serogroups and antibiotic resistance properties of Shiga-toxin producing Escherichia coli strains isolated from raw milk and traditional dairy products. Antimicrob Res Infect Control 7:53

Ranjbar R, Farsani FY, Dehkordi FS (2018b) Phenotypic analysis of antibiotic resistance and genotypic study of the vacA, cagA, iceA, oipA and babA genotypes of the Helicobacter pylori strains isolated from raw milk. Antimicrob Res Infect Control 7:115

Ranjbar R, Masoudimanesh M, Dehkordi FS, Jonaidi-Jafari N, Rahimi E (2017) Shiga (Vero)-toxin producing Escherichia coli isolated from the hospital foods; virulence factors, o-serogroups and antimicrobial resistance properties. Antimicrob Resis Infect Control 6:4

Safarpoor Dehkordi F, Barati S, Momtaz H, Hosseini Ahari SN, Nejat Dehkordi S (2013a) Comparison of shedding, and antibiotic resistance properties of listeria monocytogenes isolated from milk, feces, urine, and vaginal secretion of bovine, ovine, caprine, buffalo, and camel species in Iran. Jundishapur J Microbiol 6:284-294

Safarpoor Dehkordi F, Gandomi H, Akhondzadeh Basti A, Misaghi A, Rahimi E (2017) Phenotypic and genotypic characterization of antibiotic resistance of methicillin-resistant Staphylococcus aureus isolated from hospital food. Antimicrob Resist Infect Control 6:104

Safarpoor Dehkordi F, Haghighi N, Momtaz H, Rafsanjani MS, Momeni M (2013b) Conventional vs real-time PCR for detection of bovine herpes virus type 1 in aborted bovine, buffalo and camel foetuses. Bulgar J Vet Med 16:102"111

Safarpoor Dehkordi F, Khamesipour F, Momeni M (2014a) Brucella abortus and Brucella melitensis in Iranian bovine and buffalo semen samples: The first clinical trial on seasonal, Senile and geographical distribution using culture, Conventional and real-time polymerase chain reaction assays. Kafkas Uni Vet Fak Derg 20:821-828

Safarpoor Dehkordi F, et al. (2012) Prevalence study of theileria annulata by comparison of four diagnostic techniques in shouthwest Iran. Bulgar J Vet Med 15: 123-130

Safarpoor Dehkordi F, Valizadeh Y, Birgani T, Dehkordi K (2014b) Prevalence study of Brucella melitensis and Brucella abortus in cow's milk using dot enzyme linked immuno sorbent assay and duplex polymerase chain reaction. J Pure Appl Microbiol 8:1065-1069

Shankar H, Singh S, Singh P, Singh A, Sohal J, Greenstein R (2010) Presence, characterization, and genotype profiles of Mycobacterium avium subspecies paratuberculosis from unpasteurized individual and pooled milk, commercial pasteurized milk, and milk products in India by culture, PCR, and PCR-REA methods. Int J Infect Dis 14:e121e126

Streeter RN, Hoffsis G, Bech-Nielsen S, Shulaw W, Rings D (1995) Isolation of Mycobacterium paratuberculosis from colostrum and milk of subclinically infected cows. Am J Vet Res 56:1322-1324

Sweeney RW, Whitlock RH, Rosenberger AE (1992) Mycobacterium paratuberculosis cultured from milk and supramammary lymph nodes of infected asymptomatic cows. J Clin Microbiol 30:166-171

Szteyn J, Wiszniewska-Laszczych A, Ruszczyńska A (2008) Effectiveness of Mycobacterium paratuberculosis isolation from raw milk by means of direct isolation of DNA and classic culture. Pol J Vet Sci 11:25-28

Taylor T, Wilks C, McQueen D (1981) Isolation of Mycobacterium paratuberculosis from the milk of a cow with Johne's disease. Vet Rec 109:532-533

Tiwari A, VanLeeuwen JA, McKenna SL, Keefe GP, Barkema HW (2006) Johne's disease in Canada: Part I: Clinical symptoms, pathophysiology, diagnosis, and prevalence in dairy herds. Can Vet J 47:874-882 